Exploring Adolescent Cyber Victimization in Mobile Games: Preliminary evidence from a British cohort

Andrew K. Przybylski, $\mathrm{PhD}^{1,2}$

1. Oxford Internet Institute, University of Oxford, United Kingdom. 2. Department of Experimental Psychology, University of Oxford, United Kingdom.

Address correspondence to: Andrew K. Przybylski, 1 St. Giles' Oxford, Oxfordshire, OX1 3JS, England, andy.przybylski@oii.ox.ac.uk, +44 (0) 1865287230.

Short Title: BULLYING ON MOBILE

Key Words: adolescents; mobile gaming; electronic games; cyber victimization, cyber bullying

Funding Source: This research was supported in part by a grant from the Princess Diana Award and the British Academy.

Financial Disclosure: The author has no financial relationships relevant to this article to disclose.

Conflict of Interest: The author has no conflicts of interest to disclose.

Clinical Trial Registration: Not applicable.

Author Contributions: AKP is the sole author of this work, he designed the analytic approach, interpreted the findings and wrote the draft and final versions of the manuscript as submitted. 
BULLYING ON MOBILE

\begin{abstract}
Mobile games, those played on smartphones and tablets, are rapidly becoming a dominant form of entertainment for young people. Given most of these games provide opportunities for competitive and cooperative play they have the potential to build and enhance existing social relationships and might also provide an avenue for negative social experiences such as bullying or trolling behaviors. Unfortunately, little is understood about the prevalence or effects of cyberbullying for young people in this new form of electronic play. The present research, conducted in March 2018, surveyed a large and representative cohort of British adolescents and their caregivers $(n=2,008)$ with the aim of building an empirical understanding of this phenomenon. Adolescents were asked about their play habits and experiences and caregivers provided data on their children's socioeconomic and demographic background and psychosocial functioning. Of key interest was estimating how frequently adolescents encounter bullying in mobile games, the extent to which bullying impacts them, and the sources of support adolescents seek following these events. Results indicated that bulling in mobile games is relatively common (33.5\%) though less than one in ten experiences serious repeated bullying $(9.3 \%)$. Analysis showed males, players from a minority ethnicity, and those whose caregivers identified as having conduct problems were the most likely to report significant victimization. Further, nearly four in ten $(39.4 \%)$ reported feeling fairly or very upset by the experience and parents $(49.3 \%)$ not gaming platforms $(4.2 \%)$ where the most likely to be sought out for support after bullying was experienced. Results are discussed with respect to this soon-to-be expanding research area and recommendations for the use of open scientific methodologies are provided.
\end{abstract}

Keywords: adolescents; mobile gaming; electronic games; cyber victimization, cyber bullying 
BULLYING ON MOBILE

\section{Exploring Adolescent Cyber Victimization in Mobile Games: Preliminary evidence from a British cohort}

Bullying during childhood and adolescence is a major health problem (1). Research indicates that those who are victimized by their peers in the early years of life are at risk for multiple negative mental and physical health outcomes later in life (2). The increased availability of affordable Internetand mobile-based technologies have opened new avenues for children and adolescents to face bullying in digital contexts (3). Though much of the existing research base indicates that serious cyberbullying on social media and online games is still less common than tradition forms of face-to-face victimization (4) it remains an area of policy and research concern. Indeed, as leisure and communication move from desktop-based to mobile-based forms the potential for peer victimization through the latter medium might increase. With this concern in mind it is noteworthy that there is no nationally-representative evidence concerning the prevalence of this phenomena, how these dynamics play out on smartphones and other mobile devices, and we have little understanding of what the potential effects of victimization in this medium might have (5).

The present research was conducted with the aim of addressing these outstanding gaps in our knowledge. Specifically, this project was conducted to address three pressing research questions regarding bullying in mobile games: First, how prevalent is cyber-bullying among regular mobile gamers? Second, what demographic or psychosocial risk factors might make gamers prone to cyberbullying? Finally, what impact might cyber-bullying in mobile games have on young people and how do they respond?

\section{Methods}

\section{Participants}

A nationally representative sample of adolescents and their parents living in England, Scotland, and Wales was recruited with ICM Research using geographic data, household socioeconomic class, participant age, and gender factors based on 2011 United Kingdom Census data. This study used a 
BULLYING ON MOBILE

quota sampling approach undertaken by the polling company using the demographic factors noted above. An invitation email containing the link to the survey was mailed out to a batch of panel sample, targeting by relevant variables. The online panel had been recruited through various methods, including random via telephone sampling, random online sampling, and through active recruitment and engagement programs. Parents provided consent to participate and completed surveys first, and were asked to leave the room once they were finished so that the adolescent could complete survey responses without their presence. Parent measures lasted approximately ten minutes, and included an assessment their child's psychosocial functioning. Adolescents then provided consent and completed measures lasted approximately 15 minutes, and included those for mobile gaming, and experiences and responses to bullying in gaming contexts. Surveys also assessed variables not included in this report. The adolescent sample was evenly divided among 14 year old $(n=497)$ and 15 year old adolescents $(n=$ 507). A total of 540 participants identified as male, 461 as female, and 3 as another gender orientation. Caregiver participants were all adults. The sample was predominantly white and $8.1 \%$ of participants reporting they were from Black or minority ethnicities. Total combined household income mirrored the general population and ranged from $£ 6,500$ (1.9\%) to $£ 150,000$ or more $(2.8 \%)$. In line with best practices, all study materials, data, and code are freely available on the Open Science Framework (6).

\section{Ethical Review}

Ethical review was conducted at the author's institution prior to data collection.

\section{Measures}

Adolescents and caregivers completed the following self-report measures as part of a larger study conducted to survey the lives of British youth online in early March 2018.

Mobile Gaming. Adolescents were presented with a series of questions to determine if they were mobile gamers and estimate the extent to which they played such games. First, they were told “Many young people regularly play video games on computers (e.g. Minecraft), smartphones (e.g. Candy Crush), and gaming consoles (e.g. New Super Mario Bros.)” and were asked: “Do you play 
BULLYING ON MOBILE

video games?" Those who responded in the affirmative were asked: "Please name the games you played most in the past month and please try to be specific. For example, instead of typing "Mario Kart" or "COD" please type the specific game name, for example "Mario Kart 8" or "Call of Duty: Black Ops: Declassified". For each of these three games they were asked if they played each game on a: "Smartphone or tablet computer (e.g. iPhone, Andriod, etc.)". Results indicated a total of 184 participants had recently regularly played a mobile game for an average of $1 \mathrm{hr} 32 \mathrm{~min}(S D=1 \mathrm{hr} 22 \mathrm{~min})$ daily.

Mobile Bullying. Participants who were coded as having regularly played mobile games in the past month were told: "Here are some questions about bullying. We say a person is being bullied when another person, or a group of people, say or do nasty and unpleasant things to him or her. It is also bullying when a person is teased repeatedly in a way he or she does not like or when he or she is deliberately left out of things. Bullying can even happen over when you're playing games on your mobile. It is not bullying when a person is teased in a friendly and playful way." They were provided with a five point scale that ranged from $1=$ "I haven't been bullied in this way in the past six months" to $5=$ "Several times a week" to rate six forms of bullying in mobile games. Table 1 presents the observed frequencies of mobile bullying. In line with practices in the bullying literature a significant cut off of $\geq 2$ or 3 times a week was used to operationalize a threshold for serious bullying (7).

Psychosocial Functioning. The primary caregivers of adolescent participants completed the strengths and difficulties questionnaire, a widely used measure of adolescents' social and emotional functioning validated in community (8), academic (9), and clinical (10) settings. Caregivers provided answers on the basis their adolescent's behaviors in the past month using one of three options: "Not True" (coded 0), "Somewhat True" (coded 1) or "Certainly True" (coded 2) to rate the truth of 20 items reflecting psychosocial difficulties concerning their child, e.g., "Often fights with other children or bullies them." In line with past research (11), individual scores for peer problems, emotional problems, conduct problems, and hyperactivity, as well as an overall composite score were computed by reverse 
BULLYING ON MOBILE

scoring responses to the negatively worded statements and adding this score to the values of the positively worded ones.

Responses to Mobile Bullying. If adolescent participants reported at least one form of bullying in mobile games they were asked two follow up questions. The first concerned impact and asked: "Thinking about the LAST TIME you were bothered by something like this, how upset did you feel about it (if at all)?" using a scale that ranged from $1=$ "Very upset" to $4=$ "Not at all upset". The second was concerned with who, if anyone, they reached out for help. A list of eight contacts including "a parent or guardian" and "I reported it to the game developers (e.g. blocking, reporting player(s.)" Table 4 presents the frequencies of the emotional responses to bullying and Table 3 presents how frequently adolescents sought different kinds of support following bullying.

\section{Results}

\section{RQ1: Estimating the Prevalence of Mobile Bullying}

Frequencies presented in Table 1 indicated that mobile bullying varies widely as a function of type and severity. Overall one third of the sample (33.5\%) reported at least one form of cyber victimization in the past six months whereas nearly one in ten $(9.3 \%)$ reported significant levels (i.e. $\geq$ 2 or 3 times a week) of at least one form of bullying during this period. Overall the most prevalent form of bullying reported was being teased or being made fun of (26\%) whereas the most frequently reported form of serious bullying was the receiving unwanted messages of sexual nature $(5.6 \%)$.

\section{RQ2: Demographic and Psychosocial Correlates of Mobile Bullying}

Because many of the variables observed did not meet the assumptions of normally distributed data, non-parametric (i.e. Kendall's Tau-b) correlation analyses were used to estimate the strength, direction, and significance of the relations between the observed variables (see Table 2). Results from these analyses indicated boys tended to be the most likely to report the occurrence of any kind bullying $r(181)-.32, p<.001$, and serious bullying, $r(182)-.20, p<.001$. Non-white mobile gamers were not more likely to report any bullying $r(182)-.05, p=.537$, but were more likely to report serious bullying, 
BULLYING ON MOBILE

$r(182)-.22, p=.003$, though there were no links to household income (both $p s<.07$ ). Daily

engagement was positively related to reporting any $r(182) .18, p=.002$, and serious bullying, $r(182)$

$.27, p=.010$. Lastly, those rated by their caregivers as struggling with psychosocial difficulties were

consistently more likely to encounter both any $r(181) .18, p=.002$, and serious bullying, $r(182) .19, p$

$=.002$. Exploratory follow-up analyses indicated this result was in evidence for peer problems, conduct

problems, and emotional problems, but not for hyperactivity $(p s<.06)$. Given a number of these

factors intercorrelated and correlated with mobile bullying outcomes all seven significant predictors were entered into multiple regression models predicting any bullying and significant bullying (see

Table 3). Results from these analyses indicated that being male, daily gaming time, and emotional problems were uniquely predictive of reporting any bullying. In contrast, being male, from a minority ethnicity, and caregiver reports of adolescents' conduct problems predicted variability in significant bullying in the past six months controlling for other factors.

\section{RQ3: Emotional and Social Responses to Mobile Bullying}

Those who reported any form of bullying in mobile games were asked about how they felt about their experiences of cybervictimization and how they sought social support in response to these events. Results (see Table 4) indicated that nearly four in ten (39.4\%) of those who were bullied in mobile games said the experience made them feel very or fairly upset. Further, the data indicated (see Table 5) that adolescents were most likely to reach out to caregivers $(49.3 \%)$ and friends $(43.7 \%)$ following victimization in mobile games. By comparison, adolescents were ten times less likely to use developer reporting tools such as blocking or reporting to seek redress from offending players $(4.2 \%)$.

\section{Discussion}

Mobile games are an increasingly popular form of electronic entertainment for young people in the developed and developing world. Like other Internet-connected mediums for play and socialization they provide both potential opportunities and risks (12). This study represents the first attempt to use 
BULLYING ON MOBILE

population representative data to quantify specific risks to adolescents with respect to bullying. In line with three research questions this study materially adds to our understanding of these potential risks.

First, these findings indicate that although bullying in mobile games is relatively common in line with face to face bullying estimates $(2,4)$ serious bullying, repeated victimization at least 2 or 3 times a week is reported by fewer than one in ten mobile gamers. Though serious bullying is less prevalent, this second estimate is noteworthy as it alarmingly high when judged against the rate observed in studies of general cyberbullying which is closer to $5 \%(4,7)$. These estimates present baselines for health practitioners and game developers to target for reduction.

Second, these findings highlight that adolescents already struggling with psychosocial difficulties are the most likely to report victimisation. Though it might seem obvious, those with high levels of engagement with mobile games were also more likely report cyberbullying, there were some counter intuitive findings. First, males, not females were the more likely to experience mobile bullying. Results from multiple regression models highlighted the predictive power of gender, gaming time, and emotional problems for reporting any bullying and gender, ethnicity, and conduct problems for significant bullying. These trends run counter to what we know about gender and both cyberbullying through social media and face-to-face bullying research and suggests bullying in mobile games might be fundamentally different in some way (4). Further, null and inconsistent results for ethnicity and household income suggest these factors might not be where we should focus future research efforts.

Third, results indicate that young people may be at least temporarily emotionally impacted by bullying in mobile games. Many reported being upset by bullying in these gaming contexts. Interestingly, in response to this discomfort, existing social ties such as caregiving and peer relationships were the most likely avenues young people pursued to address bullying. In contrast, the reporting tools that game makers provide players were seldom used. This pattern of findings suggests that interventions targeted at triaging bullying in these contexts should focus on caregivers and peers as they are already trusted parties. It also indicates that longer-term improvements must be made by 
BULLYING ON MOBILE

developers so that the tools they provide are used and considered useful and trusted by adolescent gamers.

With these promising findings in mind, this research has limitations which merit note and suggest directions for future work. First, the present study examined cybervictimization at a single point in time and in isolation. Research from the face-to-face bullying literature indicates that peer victimization is often part of a larger tit for tat pattern (2). A fuller picture of the phenomenon will require collecting data on both being bullied and bullying others in mobile games over time. Second, the data under analysis were provided entirely through self-report. It is critical to augment this surveybased data with trace data provided by app and mobile game developers. If indeed reporting and blocking tools are as infrequently used as this survey indicates, it is critical to integrate insights from the gaming industry. Only then will we understand why adolescents do not use these tools and research ways to making reporting and blocking a more trusted, accessible, and effective strategy for young people.

\section{Closing Remarks}

Taken together, this study presents a useful tentative step for wider and more in-depth studies focused on specific games, affordances, and player populations. The present findings indicate that cyberbullying happens in mobile games and that players depend on existing social networks to recover from these experiences. Because the data, code, and materials are freely available this exploratory research provides a template for future work on this topic grounded in open and robust scientific practices (13). Future studies should build on what is here using transparent methodologies and preregistered trials to target interventions to limit cybervictimization. This will reduce duplicate efforts across research teams, increase the incremental value of science in the area, and ultimately help players grapple with and overcome these experiences. 
BULLYING ON MOBILE

\section{References}

1. Landstedt E, Persson S. Bullying, cyberbullying, and mental health in young people. Scand J Public Health. 2014 Jun;42(4):393-9.

2. Bowes L, Joinson C, Wolke D, Lewis G. Peer victimisation during adolescence and its impact on depression in early adulthood: prospective cohort study in the United Kingdom. BMJ. 2015 Jun 2;350:h2469.

3. Slonje R, Smith PK. Cyberbullying: another main type of bullying? Scand J Psychol. 2008 Apr;49(2):147-54.

4. Przybylski AK, Bowes L. Cyberbullying and adolescent well-being in England: a population-based cross-sectional study. Lancet Child Adolesc Health. 2017 Sep;1(1):19-26.

5. Sticca F, Perren S. Is cyberbullying worse than traditional bullying? Examining the differential roles of medium, publicity, and anonymity for the perceived severity of bullying. J Youth Adolesc. 2013 May;42(5):739-50.

6. The Authors. Study materials, data, and code. [Internet]. 2018. Available from: https://osf.io/tgby6/?view_only=e20258040e554f4c97d987f019678c4d

7. Wolke D, Lee K, Guy A. Cyberbullying: a storm in a teacup? Eur Child Adolesc Psychiatry [Internet]. 2017 Feb 10 [cited 2017 Apr 24]; Available from:

http://link.springer.com/10.1007/s00787-017-0954-6

8. Goodman R, Ford T, Simmons H, Gatward R, Meltzer H. Using the Strengths and Difficulties Questionnaire (SDQ) to screen for child psychiatric disorders in a community sample. Br J Psychiatry J Ment Sci. 2000 Dec;177:534-9.

9. Matsuishi T, Nagano M, Araki Y, Tanaka Y, Iwasaki M, Yamashita Y, et al. Scale properties of the Japanese version of the Strengths and Difficulties Questionnaire (SDQ): a study of infant and school children in community samples. Brain Dev. 2008 Jun;30(6):410-5.

10. Becker A, Hagenberg N, Roessner V, Woerner W, Rothenberger A. Evaluation of the self-reported SDQ in a clinical setting: do self-reports tell us more than ratings by adult informants? Eur Child Adolesc Psychiatry. 2004;13 Suppl 2:II17-24.

11. Van Roy B, Veenstra M, Clench-Aas J. Construct validity of the five-factor Strengths and Difficulties Questionnaire (SDQ) in pre-, early, and late adolescence. J Child Psychol Psychiatry. 2008 Dec 1;49(12):1304-12.

12. Bell V, Bishop DVM, Przybylski AK. The debate over digital technology and young people. BMJ. 2015 Aug 12;351:h3064.

13. Munafò MR, Nosek BA, Bishop DVM, Button KS, Chambers CD, Percie du Sert N, et al. A manifesto for reproducible science. Nat Hum Behav. 2017 Jan 10;1(1):0021. 
Table 1. Observed Bullying Prevalence Among Mobile Gamers

\begin{tabular}{lccccc}
\hline & Overall & \multicolumn{2}{c}{ Gender } & \multicolumn{2}{c}{ Age } \\
\cline { 2 - 6 } & Total & Boys & Girls & 14 & 15 \\
\hline $\begin{array}{l}\text { Teased or made fun of in a } \\
\text { mobile game. \% }\end{array}$ & $26.0(5.0)$ & $42.3(9.6)$ & $19.5(3.1)$ & $23.6(3.4)$ & $28.3(6.5)$ \\
$\begin{array}{l}\text { Subjected to hate speech in a } \\
\text { mobile game. \% }\end{array}$ & $16.3(3.9)$ & $31.4(7.8)$ & $10.9(2.3)$ & $17.0(2.3)$ & $16.3(5.4)$ \\
$\begin{array}{l}\text { Received threats from someone } \\
\text { in a mobile game. \% }\end{array}$ & $14.5(3.9)$ & $26.9(7.7)$ & $9.5(2.4)$ & $14.8(2.3)$ & $14.3(5.5)$ \\
$\begin{array}{l}\text { Unwanted messages of a sexual } \\
\text { nature in a mobile game. \% }\end{array}$ & $19.4(5.6)$ & $38.5(11.5)$ & $11.8(3.1)$ & $20.2(4.5)$ & $18.7(6.6)$ \\
$\begin{array}{l}\text { Have been hacked in a mobile } \\
\text { game. \% }\end{array}$ & $16.1(2.9)$ & $35.3(2.0)$ & $7.4(3.3)$ & $14.0(2.3)$ & $18.2(3.4)$ \\
$\begin{array}{l}\text { Had my personal information } \\
\text { exposed in a mobile game. \% }\end{array}$ & $12.9(3.9)$ & $24.5(9.4)$ & $8.1(1.6)$ & $12.6(4.6)$ & $13.2(3.3)$ \\
Any form of bullying. \% & $33.5(9.3)$ & $56.6(18.9)$ & $23.4(5.5)$ & $33.7(6.7)$ & $33.3(11.8)$
\end{tabular}

Note. Percentages reported in round brackets reflect serious bullying, $\geq 2$ or 3 times a week. 
Table 2. Observed Zero-Order Correlations

\begin{tabular}{|c|c|c|c|c|c|c|c|c|c|c|c|c|}
\hline & 1. & 2. & 3. & 4. & 5. & 6. & 7. & 8. & 9. & 10. & 11. & 12. \\
\hline 1. Female & -- & .020 & $-.238^{* *}$ & -.020 & -.100 & $-.235^{* *}$ & $-.247^{* *}$ & $-.224^{* *}$ & $-.305^{* *}$ & $-.351^{* *}$ & $-.224^{* *}$ & $-.321^{* *}$ \\
\hline 2. White & .020 & -- & .050 & -.100 & -.120 & -.100 & $-.179^{*}$ & $-.225^{* *}$ & -.040 & -.140 & $-.249^{* *}$ & -.050 \\
\hline 3. Household income & $-.238^{* *}$ & .050 & -- & -.050 & $-.169^{* *}$ & $.182^{* *}$ & .120 & .110 & $.130^{*}$ & $.172^{* *}$ & .100 & $.156^{*}$ \\
\hline 4. Psychosocial difficulties & -.100 & -.120 & $-.169^{* *}$ & -- & $.138^{*}$ & $.185^{* *}$ & $.245^{* *}$ & $.190^{* *}$ & $.244^{* *}$ & $.179^{* *}$ & $.282^{* *}$ & $.179^{* *}$ \\
\hline 5. Mobile gaming time & -.020 & -.100 & -.050 & $.138^{*}$ & -- & $.215^{* *}$ & $.270^{* *}$ & $.209^{* *}$ & $.148^{*}$ & $.165^{* *}$ & $.209^{* *}$ & $.188^{* *}$ \\
\hline 6. Being teased & -.140 & -.040 & .070 & .080 & .030 & -- & $.650^{* *}$ & $.619^{* *}$ & $.645^{* *}$ & $.547^{* *}$ & $.623^{* *}$ & $.831^{* *}$ \\
\hline 7. Hate speech & -.130 & $-.292^{* *}$ & -.040 & $.133^{*}$ & .120 & $.613^{* *}$ & -- & $.749^{* *}$ & $.701^{* *}$ & $.649^{* *}$ & $.891^{* *}$ & $.632^{* *}$ \\
\hline 8. Receiving threats & -.120 & $-.308^{* *}$ & .030 & .110 & $.143^{*}$ & $.481^{* *}$ & $.703^{* *}$ & -- & $.532^{* *}$ & $.660^{* *}$ & $.730^{* *}$ & $.573^{* *}$ \\
\hline 9. Sexual messages & $-.166^{*}$ & -.130 & .010 & .100 & $.171^{*}$ & $.501^{* *}$ & $.453^{* *}$ & $.578^{* *}$ & -- & $.616^{* *}$ & $.622^{* *}$ & $.695^{* *}$ \\
\hline 10. Device hacking & .040 & $-.379^{* *}$ & -.060 & $.178^{* *}$ & $.163^{*}$ & $.291^{* *}$ & $.344^{* *}$ & $.490^{* *}$ & $.270^{* *}$ & -- & $.620^{* *}$ & $.627^{* *}$ \\
\hline 11. Data exposed & $-.184^{*}$ & .050 & .090 & .010 & $.138^{*}$ & $.613^{* *}$ & $.405^{* *}$ & $.405^{* *}$ & $.453^{* *}$ & $.344^{* *}$ & -- & $.547^{* *}$ \\
\hline 12. Mobile bullying & $-.209^{* *}$ & $-.219^{* *}$ & .030 & $.191^{* *}$ & $.171^{*}$ & $.710^{* *}$ & $.623^{* *}$ & $.623^{* *}$ & $.751^{* *}$ & $.541^{* *}$ & $.623^{* *}$ & -- \\
\hline
\end{tabular}

Note. Coefficients above the diagonal reflect the presence of any bullying in the past six months whereas those below reflect serious bullying, $\geq 2$ or 3 times a week. Because bullying variables do not meet the assumptions of normality coefficients presented are Kendall's Tau-b instead of using

Pearson's method. Note coefficients marked with $\mathrm{a} *$ are significant at $p<.05$ whereas those marked with a $* *$ are significant at $p<.01$. 
Table 3. Multiple Regression Models Evaluating Unique Predictors of Mobile Bullying in the Past Six Months

\begin{tabular}{|c|c|c|c|c|c|c|c|}
\hline Outcomes & Predictors & $B$ & $S E$ & \multicolumn{2}{|c|}{$95 \% \mathrm{CI}$} & $\beta$ & $p$ \\
\hline \multirow{7}{*}{$\begin{array}{l}\text { Any bullying in } \\
\text { past six months }\end{array}$} & Female & -0.342 & 0.069 & {$[-0.478$,} & $-0.206]$ & -0.341 & $<0.001$ \\
\hline & White & 0.019 & 0.130 & {$[-0.238$} & $0.276]$ & 0.010 & 0.887 \\
\hline & Household income & -0.001 & 0.001 & {$[-0.004$,} & $0.002]$ & -0.063 & 0.354 \\
\hline & Mobile gaming time & 0.053 & 0.024 & {$[0.005$, } & $0.101]$ & 0.152 & 0.030 \\
\hline & Conduct problems & 0.012 & 0.018 & {$[-0.024$,} & $0.048]$ & 0.056 & 0.505 \\
\hline & Emotional problems & 0.050 & 0.016 & {$[0.018$,} & $0.082]$ & 0.272 & 0.002 \\
\hline & Peer problems & -0.015 & 0.017 & {$[-0.049$,} & $0.019]$ & -0.073 & 0.389 \\
\hline \multirow{7}{*}{$\begin{array}{l}\text { Significant bullying } \\
\text { in past six months }\end{array}$} & Female & -0.090 & 0.043 & {$[-0.175$,} & $-0.005]$ & -0.156 & 0.039 \\
\hline & White & -0.183 & 0.082 & {$[-0.344$,} & $-0.022]$ & -0.008 & 0.026 \\
\hline & Household income & 0.000 & 0.001 & {$[-0.002$,} & $0.002]$ & 0.062 & 0.906 \\
\hline & Mobile gaming time & 0.013 & 0.015 & {$[-0.017$} & $0.044]$ & 0.269 & 0.378 \\
\hline & Conduct problems & 0.036 & 0.011 & {$[0.014$,} & $0.058]$ & 0.047 & 0.002 \\
\hline & Emotional problems & 0.005 & 0.010 & {$[-0.015$,} & $0.025]$ & 0.051 & 0.605 \\
\hline & Peer problems & 0.006 & 0.011 & {$[-0.015$,} & $0.028]$ & -0.156 & 0.554 \\
\hline
\end{tabular}


Table 4. Emotional Reactions to Bullying in Mobile Bullying

\begin{tabular}{|c|c|c|}
\hline & $\begin{array}{c}\text { Total } \\
\text { Percentage }\end{array}$ & $\begin{array}{l}\text { Cumulative } \\
\text { Percentage }\end{array}$ \\
\hline \multicolumn{3}{|l|}{$\begin{array}{l}\text { Thinking about the LAST TIME you were bothered by something like } \\
\text { this, how upset did you feel about it (if at all)? }\end{array}$} \\
\hline $\begin{array}{ll}\text { Very upset. \% }\end{array}$ & 12.7 & 12.7 \\
\hline Fairly upset. \% & 26.8 & 39.4 \\
\hline A bit upset. $\%$ & 49.3 & 88.7 \\
\hline Not at all upset. $\%$ & 4.2 & 93.0 \\
\hline Don't know. \% & 7.0 & 100.0 \\
\hline
\end{tabular}


Table 5. Social Support Sought After Bullying in Mobile Bullying

\begin{tabular}{lrl}
\hline & \multicolumn{1}{c}{$\begin{array}{c}\text { Total } \\
\text { Percentage }\end{array}$} \\
\hline Did you contact anyone about this happening? & A parent or guardian. \% & 49.3 \\
A sibling. \% & 8.5 \\
A friend. \% & 43.7 \\
A teacher. \% & 7.0 \\
Someone whose job it is to help children (e.g. social worker, police, adviser). \% & 4.2 \\
& Another adult I trust. \% & 2.8 \\
& None of these. \% & 9.2 \\
& \\
\hline
\end{tabular}

